\title{
COMPUTER-AIDED LIGHT SHEET FLOW VISUALIZATION
}

\author{
Kathryn Stacy*, Kurt Severance**, and Brooks A. Childersi \\ NASA Langley Research Center \\ Hampton, Virginia
}

\begin{abstract}
A computer-aided flow visualization process has been developed to analyze video images acquired from rotating and translating light sheet visualization systems. The computer process integrates a mathematical model for image reconstruction, advanced computer graphics concepts, and digital image processing to provide a quantitative and visual analysis capability. The image reconstruction model, based on photogrammetry, uses knowledge of the camera and light sheet locations and orientations to project two-dimensional light sheet video images into three-dimensional space. A sophisticated computer visualization package, commonly used to analyze computational fluid dynamics (CFD) data sets, was chosen to interactively display the reconstructed light sheet images, along with the numerical surface geometry for the model or aircraft under study. A description is provided of the photogrammetric reconstruction technique, and the image processing and computer graphics techniques and equipment. Results of the computer aided process applied to both a wind tunnel translating light sheet experiment and an in-flight rotating light sheet experiment are presented. The capability to compare reconstructed experimental light sheet images and CFD solutions in the same graphics environment is also demonstrated.
\end{abstract}

\section{NOMENCLATURE}

$2 D \quad$ two-dimensional

$3 D \quad$ three-dimensional

$a_{i} \quad$ affinity distortion terms

\footnotetext{
* Computer Engineer, Member AIAA

** Computer Engineer

$\dagger$ Optical Engineer
}

Copyright (C)1993 by the American Institute of Aeronautics and Astronautics, Inc. No copyright is asserted in the United States under Title 17, U.S. Code. The U.S. Government has a royalty-free license to exercise all rights under the copyright claimed herein for Governmental purposes. All other rights are reserved by the copyright owner. $b_{i}$

$c$

$f$

$g_{i}$

$k_{i}$

$l$

M

$m$

$m_{c b}$

$n$

$n_{c b}$

$p_{i}$

$t$

$x, y, z$

$\left(x_{p}, y_{p}\right)$

$X, Y, Z$

$\alpha$

$\Delta x, \Delta y$

$\omega, \kappa, \phi$

$\Phi$

Subscripts:

C

$I$

0

$S$ coefficients of the general equation of the light sheet plane

camera constant corresponding to the lens effective focal length

input $2 D$ digital images

output $2 D$ digital images

radial distortion terms

pixel gray level value

3 by 3 rotation matrix

horizontal scan line position of a pixel

horizontal scan line position of center of brightness

column position of a pixel

column position of center of brightness

lens decentering distortion terms

parameter in parametric equations of a line in $3 D$ space

image space coordinates

photogrammetric principal point

object space coordinates

angle of attack, deg

corrections for removing biases in the imaging system

angles for camera orientation, deg

light sheet position angle, measured counterclockwise from fuselage nose, deg 


\section{INTRODUCTION}

Flow visualization techniques are important tools for the aerodynamicist. The ability to "see" a flow can aid in the development of heuristic models to describe it. One common technique used at Langley Research Center is light sheet illumination of particle-seeded flow. A single, stationary light sheet allows characteristic structures of the flow to be visualized for one slice of the three dimensional flow field. The recording camera is often restricted to a fixed location outside the test section for wind tunnel applications, or on-board the aircraft for in-flight experiments, with an oblique orientation to the light sheet. Consequently, the recorded images suffer from perspective distortion which must be accounted for when making geometric measurements. Digital image processing techniques are available to extract quantitative data from light sheet images, as well as correct for perspective distortions. These techniques rely on photographing a physical grid of known geometry in the plane of the light sheet with the recording camera. While this method is relatively simple for experiments involving stationary light sheets, it is not practical for experiments where the light sheet moves.

Advances in the design of light sheet systems have resulted in greater flexibility of the light sheet technique. For example, capabilities now exist to control the size and position of light sheets in realtime, translate and rotate single sheets through a volume, and to synchronize the video with pertinent flow oscillations ${ }^{1}$. These systems offer aerodynamicists the opportunity to see multiple slices of the $3 D$ flow field about a model. However, the task of analyzing and interpreting the recorded $2 D$ images is not an easy one. An obvious difficulty lies in trying to form a mental image of the $3 D$ flow field based on viewing a sequence of $2 D$ slices through it. Not only is visualizing the slices as a volume of data important, but also making accurate geometric measurements of features in the flow field. Clearly, it is not feasible to position a physical grid at every light sheet plane, especially for experiments where the light sheet continuously sweeps or rotates.

The purpose of this paper is to describe a computer-aided process that was developed to enhance the usefulness of $3 D$ light sheet flow visualization techniques. The main objective is to reconstruct the $3 D$ geometric shape of the visible region illuminated by translating and rotating light sheets. The computer process is a combination of digital image processing and computer graphics procedures, and the $3 D$ reconstruction algorithm based on photogrammetry. The process has been applied to light sheet images generated at Langley Research Center and provides researchers with an enhanced analysis capability for understanding flow behavior. This paper describes the sequence of procedures that have proven to be effective at providing quantitative position information for features of interest in the flow field, as well as an improved qualitative analysis capability. The description of the photogrammetric reconstruction technique, the image processing and computer graphics techniques and equipment, along with results of the computeraided process applied to both a wind tunnel and an in-flight flow visualization experiment are presented.

\section{MODEL FOR IMAGE RECONSTRUCTION}

The image reconstruction technique developed in this section is based on a simple form of photogrammetry. It replaces one of the cameras of a conventional stereo photogrammetry system with a light sheet. The reconstruction model consists of an object / image coordinate system transformation, the collinearity condition of photogrammetry, and the equation of the light sheet plane. A simple set of equations relating ideal image and object coordinates is developed in this section.

Objects and their images are located with respect to an object $(X, Y, Z)$ and image $(x, y, z)$ coordinate system, respectively. The two systems are related by a simple coordinate transformation. The origin and orientation of the object system is arbitrary. The origin of the image system is located at $\left(X_{C}, Y_{C}, Z_{C}\right)$ in object coordinates. The orientation of the image system with respect to the object system is given by the rotation angles $\omega, \phi, \kappa$ via the 3 by 3 rotation matrix $\mathbf{M}=\left(m_{i, j}\right)$ defined as

$$
\begin{aligned}
M= & {\left[\begin{array}{ccc}
\cos (\kappa) & \sin (\kappa) & 0 \\
-\sin (\kappa) & \cos (\kappa) & 0 \\
0 & 0 & 1
\end{array}\right] . } \\
& {\left[\begin{array}{ccc}
\cos (\phi) & 0 & \sin (\phi) \\
0 & 1 & 0 \\
-\sin (\phi) & 0 & \cos (\phi)
\end{array}\right] } \\
& {\left[\begin{array}{ccc}
1 & 0 & 0 \\
0 & \cos (\omega) & \sin (\omega) \\
0 & -\sin (\omega) & \cos (\omega)
\end{array}\right] . }
\end{aligned}
$$

The rotation angles are defined as positive for counter-clockwise rotation when observed from the positive end of an axis looking toward the origin. The coordinate transformation from image to object system is therefore given by

$$
\left[\begin{array}{l}
X \\
Y \\
Z
\end{array}\right]=\left[\begin{array}{lll}
m_{11} & m_{12} & m_{13} \\
m_{21} & m_{22} & m_{23} \\
m_{31} & m_{32} & m_{33}
\end{array}\right]^{T}\left[\begin{array}{l}
x \\
y \\
z
\end{array}\right]+\left[\begin{array}{l}
X_{C} \\
Y_{C} \\
Z_{C}
\end{array}\right]
$$


A typical reconstruction geometry is illustrated in figure 1 . Note that the camera lens is located at the origin of the image coordinate system, the camera points along the negative $z$-axis, and the $x$ - and $y$-axis exit to the right and top of the camera lens, respectively. The object is located at $\left(X_{O}, Y_{O}, Z_{O}\right)$ in object coordinates and its image is located at $\left(x_{I}, y_{I}, c\right)$ in image coordinates, where $c$ is the effective focal length of the lens which locates the image plane. The collinearity condition of photogrammetry requires that an object, its image, and the lens center lie on a line, as shown in the figure. A set of parametric equations for this line can be written as

$$
\begin{aligned}
& X=X_{C}+\left(X_{I}-X_{C}\right) t \\
& Y=Y_{C}+\left(Y_{I}-Y_{C}\right) t \\
& Z=Z_{C}+\left(Z_{I}-Z_{C}\right) t
\end{aligned}
$$

where $\left(X_{I}, Y_{I}, Z_{I}\right)$ are the object coordinates of the image point $\left(x_{I}, y_{I}, c\right)$, given by equation 2 . These equations can represent any point $(X, Y, Z)$ on the line. Since the object point also lies in the plane of the light sheet, its coordinates are given by the intersection of the line and an equation representing the light sheet plane. This intersection process is called triangulation. The origin of the light sheet is located at $\left(X_{s}, Y_{s}, Z_{s}\right)$, as shown in figure 1 . In general, the light sheet plane equation can be written in the form

$$
b_{1} X+b_{2} Y+b_{3} Z+b_{4}=0
$$

By substituting equation 3 into equation $4, t$ is determined to be

$$
t=\frac{b_{1} X_{C}+b_{2} Y_{C}+b_{3} Z_{C}+b_{4}}{b_{1}\left(X_{C}-X_{I}\right)+b_{2}\left(Y_{C}-Y_{I}\right)+b_{3}\left(Z_{C}-Z_{I}\right)} .
$$

By substituting this value for $t$ back into equation 3 , the unique object coordinates $\left(X_{O}, Y_{O}, Z_{O}\right)$ can be evaluated.

\section{DETERMINING MODEL INPUT PARAMETERS}

In the model described above, the parameters for the location and orientation of the camera and the light sheet are assumed known. However, these parameters need to be determined experimentally. In addition, cameras (especially short focal length cameras) can exhibit considerable image plane distortion which must be accounted for.

The equations of photogrammetry which model the projection of object coordinates $(X, Y, Z)$ to refined image plane coordinates $(x, y)$ of a camera are $^{2}$

$$
\begin{aligned}
x & =x^{\prime}+\Delta x=x_{p}-c \\
& \times\left[\frac{m_{11}\left(X_{C}-X\right)+m_{12}\left(Y_{C}-Y\right)+m_{13}\left(Z_{C}-Z\right)}{m_{31}\left(X_{C}-X\right)+m_{32}\left(Y_{C}-Y\right)+m_{33}\left(Z_{C}-Z\right)}\right] \\
y & =y^{\prime}+\Delta y=y_{p}-c \\
& \times\left[\frac{m_{21}\left(X_{C}-X\right)+m_{22}\left(Y_{C}-Y\right)+m_{23}\left(Z_{C}-Z\right)}{m_{31}\left(X_{C}-X\right)+m_{32}\left(Y_{C}-Y\right)+m_{33}\left(Z_{C}-Z\right)}\right] .
\end{aligned}
$$

The point $\left(x^{\prime}, y^{\prime}\right)$ is the measured image plane location of an object point, $\left(x_{p}, y_{p}\right)$ is the photogrammetric principle point, and the $\Delta x$ and $\Delta y$ terms represent functions for modeling the distortions of the imaging system. A commonly used set of functions for $\Delta x$ and $\Delta y$ is

$$
\begin{aligned}
& \Delta x=\bar{x}\left(k_{1} r^{2}+k_{2} r^{4}+k_{3} r^{6}\right)+p_{1}\left(r^{2}+2 \bar{x}^{2}\right) \\
& +2 p_{2} \bar{x} \bar{y} \\
& \Delta y=\bar{y}\left(k_{1} r^{2}+k_{2} r^{4}+k_{3} r^{6}\right)+2 p_{1} \bar{x} \bar{y} \\
& +p_{2}\left(r^{2}+2 \bar{y}^{2}\right)+a_{1} \bar{x}+a_{2} \bar{y}
\end{aligned}
$$

where $r^{2}=\left(x^{\prime}-x_{p}\right)^{2}+\left(y^{\prime}-y_{p}\right)^{2} \equiv \bar{x}^{2}+\bar{y}^{2}$, and $k_{i}, p_{i}$, and $a_{i}$ represent radial, lens decentering, and affinity distortion terms, respectively.

The parameters $x_{p}, y_{p}, c, k_{1}, k_{2}, k_{3}, p_{1}, p_{2}, a_{1}, a_{2}$ are determined by a photogrammetric procedure known as analytic self calibration ${ }^{3}$. This procedure is typically accomplished by bringing the recording camera into a calibration lab. The parameters $\omega, \phi, \kappa, X_{C}, Y_{C}, Z_{C}$ are determined through a technique known as space resection. Measured control points, or targets, are imaged with the camera in its proper position for obtaining light sheet images. The resection technique involves solving equation 6 above for $\omega, \phi, \kappa, X_{C}, Y_{C}, Z_{C}$ when both the image and object coordinates of the control points are known.

The technique for determining the light sheet orientation parameters $b_{i}$ differ for the experiments described below and no general analytic method has yet been incorporated. The light sheet parameters can be fixed by alignment procedures for simple geometries. In other cases transit measurements with respect to the control points must suffice. Until analytic techniques are incorporated, the light sheet parameters will limit the system accuracy.

\section{TRANSLATING LIGHT SHEET APPLICATION}

A wind tunnel laser light sheet flow visualization experiment was performed by researchers investigating the relationship between buffeting and the vortical flow field over a wing 4 . The investigation was conducted in the NASA Langley Research Center Basic Aerodynamic Research Tunnel 
(BART). The BART is a test facility dedicated to code validation ${ }^{5}$. Off-body flow visualization was obtained from laser light sheets illuminating smoke entrained in the flow. The model, shown in figure 2, consisted of twin vertical tails placed on tail support booms aft of a $76^{\circ}$ flat delta wing. The model was tested at $\alpha=20^{\circ}$ and $\alpha=30^{\circ}$.

An illustration of the BART experimental setup for $\alpha=20^{\circ}$ is shown in figure 3. A stationary video camera mounted above the test section's glass ceiling and forward of the model was used to record the experiment. The light sheet, mounted on a traversing mechanism above the test section, was directed downward onto the model. The beginning and ending light sheet locations are represented in the figure as grids. The $3 D$ region over which flow field data was obtained during the experiment is bounded by the camera field of view and the beginning and ending light sheet planes. The camera sensor array, represented by grid lines in the image plane, projects through the lens center to grid lines in the light sheet planes.

In order to obtain the position and orientation of the recording camera via the space resection procedure, a minimum of three control points are required. These points were conveniently obtained for this experiment by using the three sharp corners at the nose and wingtips of the model. The locations of these corners were obtained from model construction schematics and were assumed to be accurate. Figure 4a shows their locations in the video image. The normalized object coordinate system was defined with the origin at the model nose and the wingtips in the $X-Y$ plane, as shown in figure 5. The $X$ - coordinates of the wingtips were defined to be 100 percent model chord with the $Y-$ coordinates scaled appropriately.

Light sheet plane location data is required for the reconstruction process. The task of accurate light sheet placement was simplified for this experiment by the flat triangular model shape. The sheet was manually aligned so that both the wingtips were in the light sheet and a flat mirror placed on the model surface would reflect the light sheet back to its source. This alignment procedure insured that the light sheet was parallel to the $Y-Z$ plane. The traversing mechanism was used during the tests to position the light sheet along the $X-$ axis from 20 to 160 percent model chord, in tenpercent increments. These positions are shown in figure 5. The equations for the light sheet planes are then given by $X=0.2, X=0.3, \ldots, X=1.6$. These equations correspond to equation 4 with $b_{1}=-1.0, b_{2}=0, b_{3}=0$, and $b_{4}=0.2,0.3, \ldots, 1.6$.

A typical light sheet image for this experiment is shown in figure $4 \mathrm{~b}$. While the geometric reconstruction of the light sheet plane has been discussed in detail, the flow features revealed by scattered light and recorded as gray level variations in the image have not yet been addressed. The process of assigning a gray level value to each object point location based on the scattered light intensity of the corresponding image point will be described in the section on the computer visualization process.

\section{ROTATING LIGHT SHEET APPLICATION}

An in-flight flow visualization experiment was performed by researchers investigating the origination of the vortex system and multiple vortices over the wing of the F-106 B aircraft ${ }^{6}$. A photograph of the $\mathrm{F}-106 \mathrm{~B}$ indicating the vapor screen components is shown in figure 6 . (The figure shows a removable leading-edge flap attached to the wing which was not used for the flight data presented in this paper.) Off-surface flow visualization was obtained by illuminating propylene-glycol vapor entrained in the flow with a rotating light sheet. The propyleneglycol vapor was introduced into the flow through a probe located underneath the port wing near the leading edge. The light sheet was emitted through a narrow rotating slit located on top of the fuselage. The rotating light sheet swept out a range of angles from $40^{\circ}$ to $125^{\circ}$ aft of the aircraft centerline, and remained perpendicular to the wing upper surface. The light sheet angle was recorded during sweeps. Two video cameras, one mounted on top of the fuselage in front of the light sheet housing, and the other mounted on the port inlet, were used to record the experiment. The inlet camera had a view of the apex region and the upper wing surface, while the top camera had a view more aft along the wing upper surface.

An illustration of the F-106B experimental geometry for the top camera is shown in figure 7 . This illustration is similar to the one shown for the BART experiment except that the camera is omitted for clarity. The $3 D$ region over which flow field data was obtained is the intersection of the camera field of view and the area illuminated by the rotating light sheet. The object coordinate system shown was adopted to be compatible with other measurements performed on the aircraft. The techniques used to locate the control points and light sheet are described below.

Since no conveniently identifiable wing features were available, such as the sharp corners on the BART delta wing, the control points had to be manufactured. A video image of the control points used is shown in figure $8 \mathrm{a}$. The control points were circular pieces of cardboard centered as close as 
possible on the intersections of wing spars and ribs whose $X$ and $Y$ coordinates were documented in the original F-106B construction schematics. The $Z$ values were measured by a scale from a level line. Given the luxury of a relaxed flight schedule, a photogrammetric survey of the aircraft would have been preferred.

The light sheet was designed to rotate about a line parallel to the $Z$-axis. The measured location of the light sheet origin was $\left(X_{s}, Y_{s}, Z_{s}\right)=$ $(438.7,0.4,51.5)$ in inches as shown in figure 9 . The aircraft instrumentation system provided the rotation angle, $\Phi$, measured from the nose of the aircraft. The equation of the light sheet, as a function of $\Phi$, is given by

$$
\tan (\Phi)=\frac{\left(Y-Y_{s}\right)}{\left(X-X_{s}\right)}
$$

and equivalently in the form of Equation 4 as

$$
(\tan (\Phi)) X+(-1) Y+(0) Z+\left(Y_{s}-X_{s} \tan (\Phi)\right)=0
$$

The videotape recording of the in-flight experiment contains the primary data, i.e., vaporscattered light from the rotating light sheets. A typical image, representing one slice through the $3 D$ flow field, is shown in figure $8 \mathrm{~b}$. One factor that makes this image difficult to analyze is that the outline of the wing upper surface is barely visible. The light sheet footprint, i.e., the intersection of the light sheet with the wing upper surface, produced the most reflection and therefore appears as the brightest region in the images. The footprint provides an indication of the light sheet position when examining the $2 D$ images.

\section{COMPUTER VISUALIZATION PROCESS}

The computer visualization process is a combination of digital image processing techniques for digitizing, enhancing, and analyzing sequences of wind tunnel and in-flight images recorded on videotape, and interactive computer graphics for displaying and analyzing the reconstructed image data. All visual data analysis was performed in the Data Visualization and Animation Lab (DVAL) at Langley Research Center. The lab provides advanced visualization tools on an integrated system of highperformance graphics workstations and digital image processing equipment. DVAL resources include Silicon Graphics, Inc. (SGI) Iris workstations for performing interactive computer graphics, highquality image rendering, and advanced animations; Sun workstations for performing image analysis; and a Video Image Processing System (VIPS) for performing video-rate image acquisition, processing, and storage. The VIPS is a combination of hardware and software designed to provide a specialized capability for applying enhancements at videorates to sequences of wind tunnel or in-flight images recorded on videotape.

The overall computer process is illustrated in figure 10. Videotape frames were digitized and processed on the VIPS. The digital images were then analyzed on the Sun computer to extract vortex core locations. The images and the core locations were subsequently transferred to an Iris workstation where the triangulation software was executed to map image pixels into $3 D$ space. The camera and light sheet parameters are necessary inputs to the triangulation software. The Flow Analysis Software Toolkit (FAST) on the Iris was used to interactively display the $3 D$ image data along with the numerical surface geometry of the model.

\section{Digitizing and Processing the Videotape}

In the two examples described above, the visual record of the light sheet flow visualization experiment is a standard S-VHS videotape. Prior to image reconstruction, these analog video signals must be converted to digital images. The VIPS, composed of an Imaging Technologies, Inc. (ITI) Series 150 modular image processing subsystem and a Storage Concepts, Inc. real-time digital disk (RTDD) subsystem interfaced to a Sun computer, was used to digitize, process, and store select frames from the raw video footage of the flow visualization experiments. The block diagram in figure 11 illustrates the pipeline configuration of the modules within the Series 150 subsystem and the interface to the RTDD subsystem. The Analog-Digital Interface (ADI) converts the analog video signal into a twodimensional array of numbers that can be passed sequentially through the pipeline to the other modules in the subsystem. For each frame of video, the ADI divides 480 of the active horizontal video lines into 512 samples, known as pixels. Each pixel location is assigned an integer gray level value between 0 and 255 corresponding to the brightness of the image at that location. The resulting $512 \times 480 \times$ 8 bit digital images are passed through the pipeline to other modules in the pipeline where additional processing may be performed. The Real-Time Modular Processor (RTMP) is the first module in the pipeline, followed by the Arithmetic Logic Unit (ALU), and finally the Frame Buffer (FB). Pixels are processed through the pipeline at video-rates, or 30 frames per second. The RTDD is capable of storing approximately eight minutes of full-frame 
digital image data. The software interface is Noesis Vision, Inc's VISILOG Image Processing Software.

During the BART experiment, the light sheet was positioned at 15 different stations along the model, ranging from 20 percent model chord to 160 percent model chord, as shown in figure 5 . Approximately 20 seconds of video was recorded for each light sheet station. The ITI pipeline was configured to process the digital images coming from the ADI using a pointwise recursive digital filter of the form:

$$
g_{i}=a f_{i}+(1-a) g_{i-1}
$$

where $f_{i}$ and $g_{i}$ are the input and output images, respectively, and $0<a<1$. This temporal digital filter is commonly used in signal processing ${ }^{7}$ to smooth digital data. A weight of $a=0.25$ was selected with the goal of removing noise in the images without significantly altering temporal information. The first input image was $f_{0}$ and an image of all zeros was used as the initial value for $g_{-1}$. The resulting time-filtered digital images were stored on the RTDD. The processed images were then played back from the RTDD at speeds less than video rates for inspection. The researcher selected one representative image for each light sheet station based on subjective image quality. The images shown in figure 4 are two examples of images digitized and processed on the ITI subsystem.

For the F-106B in-flight experiment, the researcher previewed the videotape in advance, and selected specific frames to be digitized. Frames of interest were identified by the visual time code appearing on each video frame. No processing was performed on these images prior to image reconstruction.

\section{Extracting Vortex Core Locations in $2 D$ Images}

Once the light sheet images have been digitized, interactive image analysis techniques can be applied in order to extract quantitative information such as vortex core locations. The BART light sheet image at station $X=1.1$ will be used as an example. An enlarged gray scale image of the port side vortex is shown in figure $12 \mathrm{a}$. The vortex core appears as a dark interior region, void of seeding particles. The location of the vortex core centroid is determined by finding the center of mass of the pixels within these dark regions. A better terminology might be center of brightness, since a brightness value is more commonly associated with pixels. The horizontal scan line and column position of the center of brightness is denoted by $\left(m_{c b}, n_{c b}\right)$. This coordinate is determined from $N$ pixels having gray level values $l_{1}, l_{2}, \ldots, l_{N}$, and positions $\left(m_{1}, n_{1}\right),\left(m_{2}, n_{2}\right), \ldots,\left(m_{N}, n_{N}\right)$ by

$$
m_{c b}=\frac{\sum_{i=1}^{N} m_{i} l_{i}}{\sum_{i=1}^{N} l_{i}} \text { and } n_{c b}=\frac{\sum_{i=1}^{N} n_{i} l_{i}}{\sum_{i=1}^{N} l_{i}}
$$

Since the center of a dark region is of interest, the gray level values of the pixels in this region are contrast reversed, i.e., replaced with $255-l_{i}$, for the centroid computation. The $m_{c b}$ and $n_{c b}$ coordinates are the vortex core centroid.

The center of brightness computation is restricted to a subset of pixels comprising the dark interior region of the vortex. This subset is determined using a sequence of simple steps. First, the researcher specifies an initial estimate of the vortex core location by selecting a single pixel somewhere inside the dark interior region of the vortex. This initial guess pixel serves as the input to a technique which finds the valley of the region surrounding this pixel, analogous to letting a ball roll down a hill.

The output pixel location replaces the researcher's initial guess and serves as the input to a technique which searchs for the ridge of the hill. The result is a closed boundary, shown in figure $12 \mathrm{~b}$, defining the subset, A, of pixels to be considered for use in the center of brightness computation. Pseudocolor, an assignment of colors to gray level values, is used in the figure to emphasize contrast variations within the vortex core. Once A has been determined, B, a subset of $\mathrm{A}$, is defined by applying a gray level threshold to the pixels in A. Subset B, shown in Figure 12c as an entirely black region, represents the pixels in subset $\mathrm{A}$ with a gray level value below the chosen threshold. The pixels in B are used to compute $m_{c b}$ and $n_{c b}$ from the equations above, after contrast reversal. The crosshair in Figure $12 \mathrm{c}$ indicates the computed pixel location of the vortex core.

\section{Interactive Display of $3 D$ Data}

The photogrammetric reconstruction of one $512 \times 480$ digital image yields a $3 D$ coordinate and a gray level value for every pixel, requiring 7.5 Mbytes of storage space. The total storage requirement depends on the number of light sheet planes to be analyzed and the additional storage required for the numerical surface geometry of the model. Typically, anywhere between 10 and 15 images per test condition were studied for the two experiments discussed above. A sophisticated visualization package is required to interactively display experimental data sets of this magnitude. The package chosen for this work is the Flow Analysis Software Toolkit (FAST) running on the Iris workstation. FAST was 
originally developed as a post-processing graphics environment for viewing and analyzing large $3 D$ computational fluid dynamics (CFD) databases 8 . The package allows researchers to load in CFD grids and solution data sets stored on the Iris in PLOT3D format ${ }^{9}$, and to visualize this data. In this work, experimental data is stored much the same way CFD data sets are. A data set containing the $3 D$ coordinates of object points corresponding to video image points is output by the triangulation software. This data set is also a PLOT3D grid, and can therefore be loaded into FAST in the same manner a CFD grid is loaded. A data set containing image intensities is output during the digitization process. This data set can be thought of as containing the functions values for each grid location, and can also be loaded into FAST in much the same way a CFD solution data set is loaded.

There are many features of the highly interactive FAST environment that are useful for analyzing the experimental data sets. Figure 13 shows several user interface windows that appear on the Iris display during a FAST session. The delta wing numerical surface geometry, reconstructed light sheet images, and vortex core paths are shown in the $A c$ tive View Window. In order to create this display, grid files for the delta wing, twin tails, light sheet stations, and vortex core locations are selected for data input. Also, the light sheet intensities corresponding to the light sheet grid are selected. Pseudocolor is used to display the light sheet intensities. A range of low intensities, (from 0 to 20) are clipped, i.e., not displayed, using a thresholding option in FAST. This allows only the vortex structure to be seen. Also, object points with $Z$ values less than 0 are not displayed, eliminating unimportant data below the wing. The entire scene can be rotated, translated, and zoomed in and out using the interactive viewing controls. A data probe and probe typeout can be used to examine the $3 \mathrm{D}$ coordinates of any grid point in any of the scene objects. In the figure, the probe is being used to extract the $X, Y, Z$ value for the port side vortex core at light sheet station $X=0.7$.

The entire scene shown in the Active View Window in figure 13 is created in a progression, as shown in figure 14a-f. First, the numerical surface geometry of the delta wing and left and right tails is read in and displayed with grid lines (figure 14a). The model can also be rendered as a smooth shaded surface to provide a more realistic view (figure 14b). Figure 14c shows the smooth shaded surface with the light sheet grid for station $X=1.1$. The function values at each grid point, in this case image intensities, are interpolated to produce a smooth shaded surface as shown in figure $14 \mathrm{~d}$. Function values below a specified value are clipped and every other light sheet station is displayed in figure $14 \mathrm{e}$. Lastly, figure $14 \mathrm{f}$ shows a continuous vortex core path by connecting the vortex core points extracted for each light sheet station with straight lines. A similar progression for the $\mathrm{F}$ $106 \mathrm{~B}$ port wing is shown in figure $15 \mathrm{a}-\mathrm{f}$. The entire F-106B aircraft can be displayed in FAST with selected light sheet images as shown in figure 16 . Details of the F-106B aircraft, such as the nose, the fuselage, the light sheet housing, and the light sheet are accentuated in this view.

The objects in a scene can be rotated and viewed from any angle. Figure 17 shows a top and side view of the delta wing with just the reconstructed vortex core points, connected by straight lines. The top view gives insight into the distance the core path is from the leading edge, whereas the side view provides insight into the height of the vortex core above the delta wing surface.

An advantage of using FAST to analyze the experimental data sets is the capability to perform visual comparisons with CFD data sets. Figure 18 shows the delta wing with experimental data on the port side and computational stagnation pressure contours on the starboard side ${ }^{10}$. The computed yellow particle traces indicating the path of the vortex core show good correlation with the experimental results.

\section{CONCLUSIONS}

It has been demonstrated that the computeraided image reconstruction technique can be effectively applied to research projects using moving light sheets to visualize $3 D$ flow fields. The technique provides researchers with a new $3 D$ quantitative analysis capability for $2 D$ light sheet images recorded on videotape that were previously difficult to evaluate. Once represented in $3 D$, the images can be observed from any viewpoint, freeing the researcher from the original camera view. In addition to a powerful $3 D$ visualization tool, the technique provides mathematically precise quantitative information about locations and structures of vortex systems. FAST allows the $X, Y, Z$ locations and function values of image points and model grid points to be examined interactively. Information, such as the distance from vortex core locations to the wing leading edge, is easily extracted. This technique also shows promise in aiding the visual comparison of experimental and CFD data. Experimental data is visualized using the same computer graphics environment, FAST, commonly used for analyzing and interpreting CFD data. 


\section{$\underline{\text { ACKNOWLEDGMENTS }}$}

Special thanks go to Brent Bates for supplying the F-106B numerical surface geometry and Sherri Krist for supplying the twin tail delta wing numerical surface geometry. Also, thanks go to Christine Matthews, Elizabeth Avis, Patricia Wright, and Blake Williams for the support they provided with the VIPS system and software development.

\section{REFERENCES}

1. Rhodes, David B.; Franke, John M.; Jones, Stephen B.; and Leighty, Bradley D.: A TwinMirrored Galvanometer Laser Light Sheet Generator. NASA TM 100587, June 1988.

2. Karara, H. M., Editor in Chief: Non- Topographic Photogrammetry, 2nd Edition. American Society for Photogrammetry and Remote Sensing, Falls Church, Virginia, 1989.

3. Snow, Walter L.; Childers, Brooks A.; and Shortis, Mark R.: The Calibration of Video Cameras for Quantitative Measurements. 1993 ASD/TMD Proceedings, 39th Annual Instrumentation Symposium, Instrument Society of America (Albuquerque, New Mexico), May 2-6, 1993.

4. Washburn, Anthony E.; Jenkins, Luther N.; and Ferman Marty A.: Experimental Investigation of Vortex-Fin Interaction. AIAA-93-0050, January 1993.

5. Sellers, William L.,III; and Kjelgaard, Scott O.: The Basic Aerodynamics Research Tunnel - A Facility Dedicated to Code Validation. AIAA-881997, May 1988.

6. Lamar, John E.; Brandon, Jay; Johnson, Thomas D., Jr.: Vortex Features of F-106B Aircraft at Subsonic Speeds. AIAA Paper No. 933471; To be presented at AIAA 11th Applied Aerodynamics Conference in Monterey, CA, August 1993.

7. Hamming, Richard W.; Digital Filters, Second Edition. Prentice-Hall, 1983.

8. Walatka, Pamela P; Clucas, Jean; McCabe, R. Kevin; Plessel, Todd; andPotter, Rick: FAST 1.0 User Guide. NASA Ames Research Center RND-92-015, November 1992.

9. Walatka, Pamela P.; Buning, Pieter G.; Pierce, Larry; and Elson, Patricia A.: PLOT3D User's Manual. NASA TM 101067, March 1990.

10. Krist, Sherri; Washburn, Anthony; and Visser, Kenneth D.: A Computational and Experimental Investigation of a Delta Wing with Vertical Tails. AIA A-93-3009, July 1993. 